\title{
SECOND ORDER NONLINEAR OSCILLATIONS
}

\author{
BY C. V. COFFMAN ${ }^{1}$ AND J. S. W. WONG
}

Communicated by Fred Brauer, June 4, 1969

We are concerned here with the oscillatory behavior of solutions of the following second order nonlinear ordinary differential equation

$$
y^{\prime \prime}+y F\left(y^{2}, x\right)=0, \quad x>0,
$$

where $y F\left(y^{2}, x\right)$ is continuous for $x>0$ and $|y|<\infty$, and $F(t, x)$ is nonnegative for $x>0$ and $t \geqq 0$. The prototype of equation (1) is the so-called generalized Emden-Fowler equation:

$$
y^{\prime \prime}+q(x)|y|^{\gamma} \operatorname{sgn} y=0, \quad x>0,
$$

where $q(x) \geqq 0$ and $\gamma>0$. Here we are interested in the determination of sufficient conditions for the existence of at least one oscillatory solution, and also in the complementary problem, i.e., the determination of conditions which guarantee the nonexistence of oscillatory solutions of (1).

The problem of oscillation and nonoscillation of solutions of equations (1) and (2) has been of great interest in recent years; we refer the reader to [7] for a current survey of the literature on this subject. In most of these studies, it is convenient to subdivide the discussion according to the nonlinear character of equation (2). We say that equation (2) is in the sublinear case if $\gamma \leqq 1$ and it is in the superlinear case if $\gamma \geqq 1$. More generally, one says that equation (1) is in the sublinear case if $F(t, x)$ is monotone decreasing in $t$ and it is in the superlinear case if $F(t, x)$ is monotone nondecreasing in $t$. Results in the same direction as ours may be found in Belohorec [1] for equation (2) when $\gamma<1$, and in Jasny [3], Kurzweil [5], Kiguradze [4], and Nehari [6] for equation (2) when $\gamma>1$. For the more general equation (1), the superlinear case has been considered in Nehari [6], and in Coffman and Wong [2], but as far as we know the corresponding sublinear case has not been investigated. In the present work, we attempt to present a unified treatment for the study of this specific oscillation problem both in the sublinear and the superlinear case. The main results include oscillation and nonoscillation theorems for both of the two classes of equation (1) and contain as special cases all of the results cited above. In fact, in the process of this generalization

1 The research of the first-named author was supported by NSF GP-7662. 
we also achieve a certain degree of simplification. The basic method of proof depends upon the introduction of certain "Lyapunov" type energy functions and comparison with an appropriate oscillatory or nonoscillatory second order linear equation.

Let $G(t, x)=\int_{0}^{t} F(s, x) d s$, and let $p(x)$ be a positive continuous function on $(0, \infty)$ such that the linear equation $z^{\prime \prime}+p(x) z=0$ is nonoscillatory. The main results in the sublinear case are

Theorem 1. Assume that (1) is sublinear and that

(a) there exists a $\lambda>0$ such that $z^{\prime \prime}+(1+\lambda) p(x) z=0$ is oscillatory,

(b) there exist positive functions $\omega \in C^{2}[0, \infty), \psi \in C^{3}[0, \infty)$ such that

(i) $\omega^{\prime} \leqq 0$,

(ii) $\psi$ is a solution of $z^{\prime \prime}+p(x) z=0$,

(iii) $\omega^{2}\left(\psi^{2}\right)^{\prime \prime \prime}+\left(\omega^{2}\right)^{\prime} \psi \psi^{\prime \prime} \leqq 0$,

(iv) $-\omega^{2} \psi^{3} \psi^{\prime \prime} \geqq c>0$,

(c) there exist $M_{0}, x_{0}>0$ such that $F(t, x) \geqq(1+\lambda) p(x)$ for $x \geqq x_{0}$ and $t \leqq M_{0} \psi^{2}(x)$,

(d) the function $\omega^{2} \psi^{2} G\left(\alpha^{2} \psi^{2}, x\right)$ is nonincreasing in $x$ for every $\alpha>0$. Then (1) has an oscillatory solution.

Theorem 2. Assume that (1) is sublinear and that

(a) $F(t, x)$ satisfies $y_{1} F\left(y_{1}^{2}, x\right) \leqq y_{2} F\left(y_{2}^{2}, x\right)$ for $y_{2} \geqq y_{1}$,

(b) there exists a positive function $\psi \in C^{3}(0, \infty)$ such that $\omega(x)$ $=\int^{x} d \sigma / \psi^{2}(\sigma) \rightarrow \infty$ as $x \rightarrow \infty$ and $\omega^{2}\left(\psi^{2}\right)^{\prime \prime \prime}+\left(\omega^{2}\right)^{\prime} \psi \psi^{\prime \prime} \geqq 0$,

(c) for every $\alpha>0, \omega^{2} \psi^{2} G\left(\alpha^{2} \psi^{2}, x\right)$ is nondecreasing and bounded above.

Then equation (1) is nonoscillatory.

Taking $p(x)=1 / 4 x^{2}, \omega(x) \equiv 1, \psi(x)=x^{1 / 2}$ in Theorem 1 , we obtain the result of Belohorec [1, Theorem 5] for equation (2) with $\gamma<1$. Similarly, choosing $p(x)=(1-\epsilon)^{2} / 4 x^{2}, \omega(x)=x^{\epsilon}, \psi(x)=x^{(1-\epsilon) / 2}, 0<\epsilon$ $<1$, in Theorem 2, we obtain another result of Belohorec [1, Theorem 6]. In fact, these specializations yield genuine extensions of Belohorec's result to the more general equation (1). On the other hand, we may choose different admissible functions $p(x), \omega(x)$ and $\psi(x)$ to obtain

Corollary. Let $0<\gamma<1$.

(a) If $q(x) x^{(\gamma+3) / 2}(\log x)^{\beta}$ is nonincreasing for some $\beta \leq 0$ and bounded away from 0 , then equation (2) has an oscillatory solution;

(b) If $q(x) x^{(\gamma+3) / 2}(\log x)^{\beta}$ is nondecreasing for some $\beta>(2-\gamma) / 2$ and bounded above, then equation (2) is nonoscillatory. 
The corresponding results for the superlinear case are

THEOREM 3. Assume that (1) is superlinear and that

(a) there exists a $\lambda>0$ such that $z^{\prime \prime}+(1+\lambda) p(x) z=0$ is oscillatory,

(b) there exists a positive function $\psi \in C^{3}[0, \infty)$ such that

(i) $\psi$ is a solution of $z^{\prime \prime}+p(x) z=0$,

(ii) $\int^{\infty} d x / \psi^{2}(x)=\infty$,

(iii) $\left(\psi^{2}\right)^{\prime \prime \prime} \geqq 0$,

(iv) $-\psi^{3} \psi^{\prime \prime} \leqq K<\infty$.

(c) there exist $M_{0}, x_{0}>0$ and $\lambda^{\prime}>\lambda$ such that $F(t, x) \geqq\left(1+\lambda^{\prime}\right) p(x)$, for $x \geqq x_{0}$ and $t \geqq M_{0}^{2} \psi^{2}(x)$.

(d) the function $\psi^{2} G\left(\alpha \psi^{2}, x\right)$ is nondecreasing in $\psi$ for every $\alpha>0$. Then equation (1) has an oscillatory solution.

TheOREM 4. Assume that (1) is superlinear and that

(a) there exist positive functions $\phi \in C^{2}(0, \infty)$ and $\psi \in C^{3}(0, \infty)$ such that

(i) $\phi$ is a solution of $z^{\prime \prime}+p(x) z=0$,

(ii) $\left(\psi^{2}\right)^{\prime \prime \prime} \leqq 0$,

(iii) $\phi=o(\psi)$, as $x \rightarrow \infty$,

(iv) $\phi^{2}\left(\psi^{2}\right)^{\prime \prime} \geqq c>0$,

(v) $\phi^{3} \phi^{\prime \prime} \leqq K<\infty$.

(b) For every $\alpha>0, \psi^{2} G\left(\alpha \psi^{2}, x\right)$ is nonincreasing in $x$. Then every solution $y(x)$ of (1) satisfying $\int^{\infty}\left|y\left(\phi^{2}\right)^{\prime \prime}\right|<\infty$ must be nonoscillatory.

Now if we take $p(x)=1 / 4 x^{2}, \psi(x)=x^{1 / 2}$ in Theorem 3 , we obtain our earlier oscillation result for equation (1), [2, Theorem 1]. Again if we take $p(x)=1 / 4 x^{2}, \phi(x)=x^{1 / 2} \psi(x)=(x \log x)^{1 / 2}$ in Theorem 4 , we obtain the nonoscillation result given in [2, Theorem 2]. Further specializing our results to equation (2), we obtain the results of Jasny [3], Kurzweil [5], Kiguradze [4], and Nehari [6] cited above. Full statements with proofs of the above theorems as well as other details will appear elsewhere.

\section{REFERENCES}

1. S. Belohorec, On some properties of the equation $y^{\prime \prime}(x)+f(x) y^{\alpha}(x)=0$, Mat. Casopis Sloven. Akad. Vied. 17 (1967), 10-19.

2. C. V. Coffman and J. S. W. Wong, On a second order nonlinear oscillation problem, Trans. Amer. Math. Soc. (to appear).

3. M. Jasny, On the existence of an oscillatory solution of the nonlinear differential equation of the second order $y^{\prime \prime}+f(x) y^{2 n-1}=0, f(x)>0$, Casopis Perst. Mat. 85 (1960), 78-83. 
4. I. T. Kiguradze, On conditions for oscillation of solutions of the equation $u^{\prime \prime}$ $+a(t)|u| \operatorname{sgn} u=0$, Casopis Pést. Mat. 87 (1962), 492-495.

5. J. Kurzweil, $A$ note on oscillatory solution of equation $y^{\prime \prime}+f(x) y^{2 n-1}=0$, Casopis Pest. Mat. 85 (1960), 357-358.

6. Z. Nehari, A nonlinear oscillation problem, J. Differential Equation 5 (1969), $452-460$.

7. J. S. W. Wong, On second order nonlinear oscillation, Funkcial. Ekvac. 11 (1969), 207-234.

Carnegie Mellon University. Pittsburgh, Pennsylvania 15213 